SANDIA REPORT

SAND96-1030 - UC-905

Unlimited Release

Printed May 1996

$$
\begin{array}{r}
\text { PEDER } \\
\text { MN } 1595
\end{array}
$$

\title{
Lessons-Learned from the Document Production System Redevelopment (DPS/R)
}

\author{
M. J. Rouse, R. R. Baird, D. G. Heckart
}

\section{Prepared by}

Sandia National Laboratories

Albuquerque, New Mexico 87185 and Livermore, California 94550

for the United States Department of Energy

under Contract DE-AC04-94AL85000

Approved for public release; distribution is unlimited.

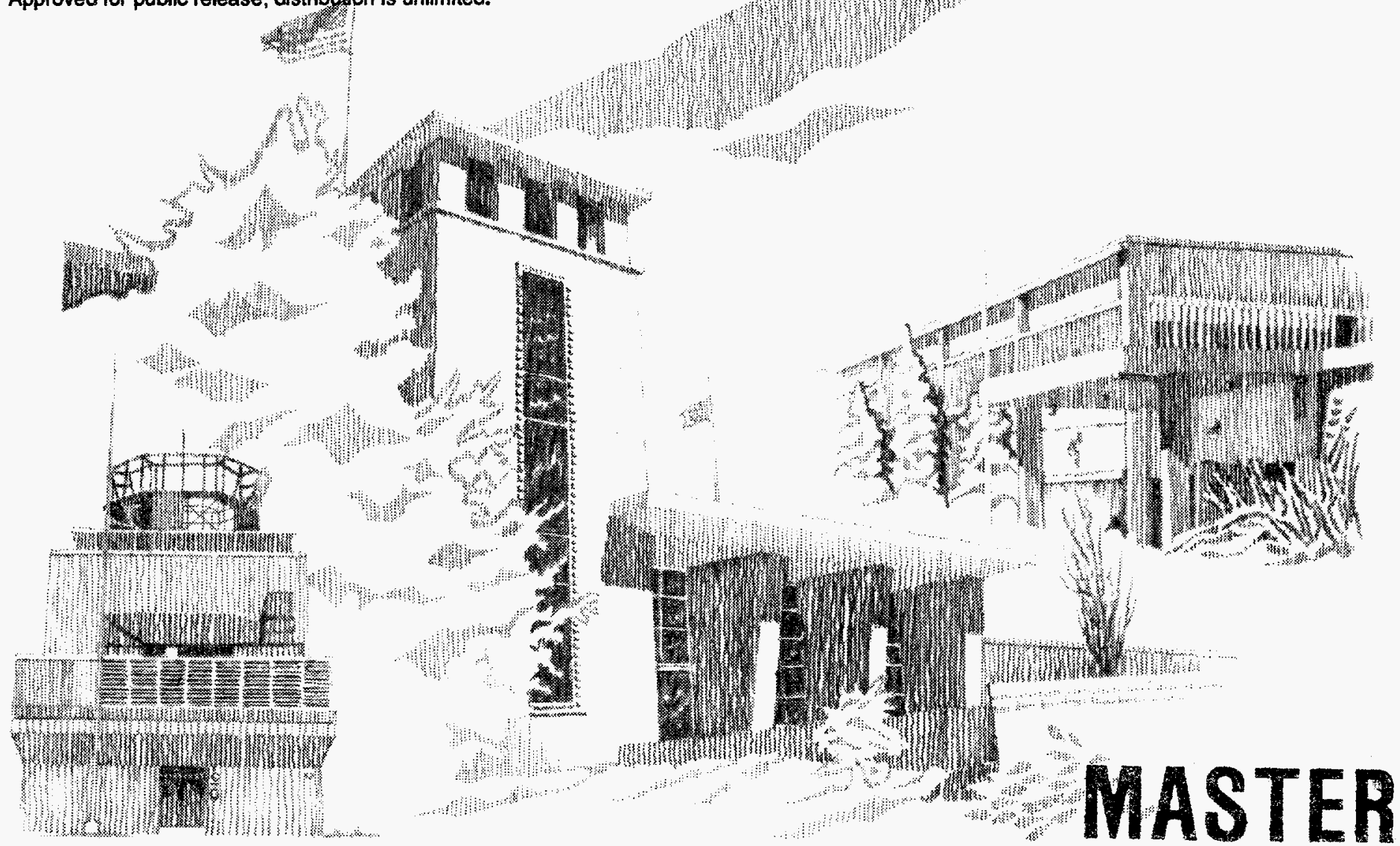


Issued by Sandia National Laboratories, operated for the United States Department of Energy by Sandia Corporation.

NOTICE: This report was prepared as an account of work sponsored by an agency of the United States Government. Neither the United States Government nor any agency thereof, nor any of their employees, nor any of their contractors, subcontractors, or their employees, makes any warranty, express or implied, or assumes any legal liability or responsibility for the accuracy, completeness, or usefulness of any information, apparatus, product, or process disclosed, or represents that its use would not infringe privately owned rights. Reference herein to any specific commercial product, process, or service by trade name, trademark, manufacturer, or otherwise, does not necessarily constitute or imply its endorsement, recommendation, or favoring by the United States Government, any agency thereof or any of their contractors or subcontractors. The views and opinions expressed herein do not necessarily state or reflect those of the United States Government, any agency thereof or any of their contractors.

Printed in the United States of America. This report has been reproduced directly from the best available copy.

Available to DOE and DOE contractors from

Office of Scientific and Technical Information

PO Box 62

Oak Ridge, TN 37831

Prices available from (615) 576-8401, FTS 626-8401

Available to the public from

National Technical Information Service

US Department of Commerce

5285 Port Royal Rd

Springfield, VA 22161

NTIS price codes

Printed copy: $\mathrm{A03}$

Microfiche copy: A01 


\section{DISCLAMMER}

Portions of this document may be illegible in electronic image products. Images are produced from the best available original document. 
SAND96-1030

Distribution

Unlimited Release

Printed May 1996

Category UC-905

\title{
Lessons-Learned from the Document Production System Redevelopment (DPS/R)
}

\author{
M. J. Rouse, R. R. Baird, and D. G. Heckart \\ Procurement Center \\ Information Systems and Technology Department \\ Sandia National Laboratories \\ Albuquerque, NM 87185
}

\begin{abstract}
Increased complexity of the computing environment is probably the most universal observation of developers going into Client/Server computing. The Lessons Learned document Provides some condensed bits of expertise gained by the Document Production System Redevelopment team. The intent is to distribute in a simplified format the knowledge we have gained, and to promote discussion of certain areas having unresolved or less-than-perfect resolution. Included are first, a description of the hardware and software used. The lists of hardware and software are a good basic guide for the components of a robust data server and provide a framework for subsequent discussion of technical points learned during the development. The second part of the document is a discussion of fine points about Novell, Sybase and Visual Basic as applied to the DPS/R development. Areas covered include the hardware of the database server, Novell operating system, Sysbase database tools and Visual Basic.
\end{abstract}


Intentionally Left Blank 


\section{Contents}

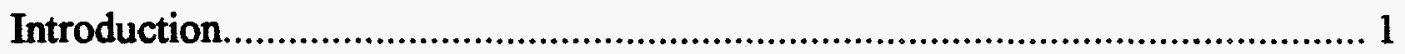

Summary Description of the System and Database .............................................. 1

Document Production Summary Description .............................................. 1

Hardware/Software Environment for DPS/R ........................................ 2

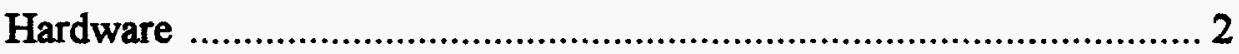

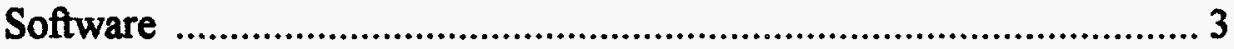

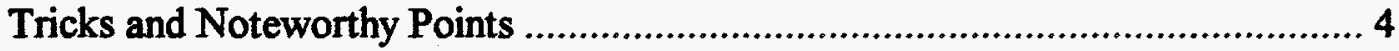

About Sybase

Process for Getting All Customers off the Database ............................... 4

Most Used Novell Commands and Sybase Instructions ........................... 5

Memory and Disk Management ..................................................... 5

Running Out of Log Disk Space ........................................................ 6

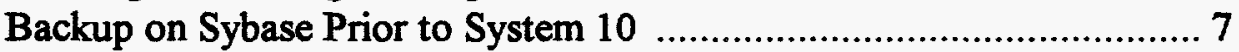

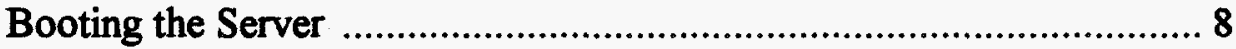

About Novell

Special Caution About Net-Prot Virus Scanner …….......................... 8

To Use a CD ROM on a Novell Machine ........................................ 9

Sample AUTOEXEC.NCF for IBM Server Using SCSI,

LANstreamer, Token-Ring ................................................... 9

Autoexec.NCF Installation Situations .......................................... 13

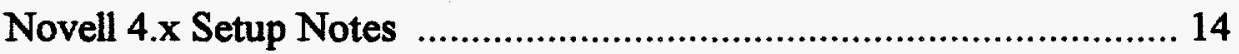

Login Scripts - a Primer ................................................................. 16

Notes on Recovery when Novell Will Not Boot ................................. 17

Data Communications Under Novell and TCPIP ............................. 18

About Visual Basic

Date-Time Selects Using VB and Sybase......................................... 19

SYBASE Refuses to Open Table ................................................. 19

MousePointer Tip ........................................................................... 19

LAN Addressing -- Router Considerations for Sybase ...................... 19

Desktop DBA TimeOut Due to DB Size ........................................ 20

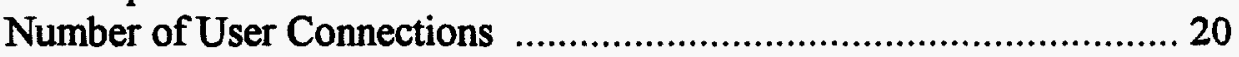

DOS BCPs and Date Field Problems .............................................. 20

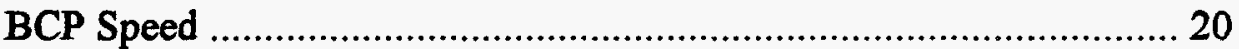

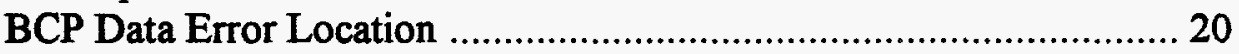

Sybase Sensitive to Com-Line Noise _............................................. 20

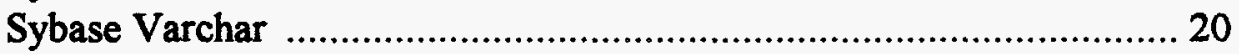

Sybase DAT Tape Backup ............................................................ 21

Subroutine Calls from Dynamic Lists .............................................. 21

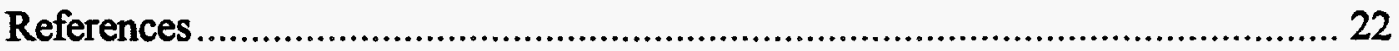




\section{Intentionally Left Blank}




\section{INTRODUCTION}

The following document presents some of the experiences of the Document Production System Redevelopment team. The intent is to distribute the knowledge we have gained, and to promote discussion of certain areas having unresolved or less-than-perfect resolution.

\section{SUMMARY DESCRIPTION OF THE SYSTEM AND DATABASE}

This section of the document provides a summary description of 1) the Document Production System, and 2) the hardware and software tools used in the redevelopment.

\section{DOCUMENT PRODUCTION SUMMARY DESCRIPTION}

Procurement processes annually about 16,000 actions for acquisitions requiring a contract document. A little more than half of the actions are amendments. The other half consists of Requests for Quote or Proposal, awarded competitive procurements and Sole Source contracts. Procurement also processes annually some 25,000 to 30,000 low value procurements. In June 1995, all procurements requiring a contract document began processing through the new Document Production Replacement (DPS/R). In early 1996 the low value procurements will be processed by DPS/R.

The focus of the DPS/R project is to bring modern Graphical User Interface, robust database tools and comprehensive application coding tools to the Procurement process. The application accomplishes several process improvements including:

- Places a central point of data collection directly in the hands of buyers

- Enforces business rules pertaining to procurement data

- Enforces financial rules for allocation of funds from requisitions

- Enforces rules for clauses containing details of contractor performance as required by Legal, DOE and Sandia policy

- Tracks versions of certain clause documents sent to suppliers with instructions directing the supplier to retain the document and refer to the document for future transactions with Sandia

- Automates the production of documents, eliminating clerical support for document preparation, typing and data transfer to the corporate data base

- Incorporates support for Microsoft Word 6 allowing statements of work prepared by line-organization customers (including graphics for equations and pictures) to be moved directly into the procurement document

- Interfaces to HRIS, Financial, and historic Procurement and supplier data residing on the LIS

Provides an automated fax capability to eliminate clerical support required in manual faxing. 


\section{HARDWARE/SOFTWARE ENVIRONMENT FOR DPS/R}

\section{Hardware.}

The DPS/R production database server is an IBM PS/2 model 95 server with a $60 \mathrm{Mhz}$ Intel Pentium chip, 112MB RAM, 9GB of disk space, a 4mm tape backup, IBM AutoLANstreamer network cards, and Novell 3.12. Sybase version 4.2 was then installed on this machine for exclusive use for DPS/R database use.

The controllers and drives were initially set up as follows:

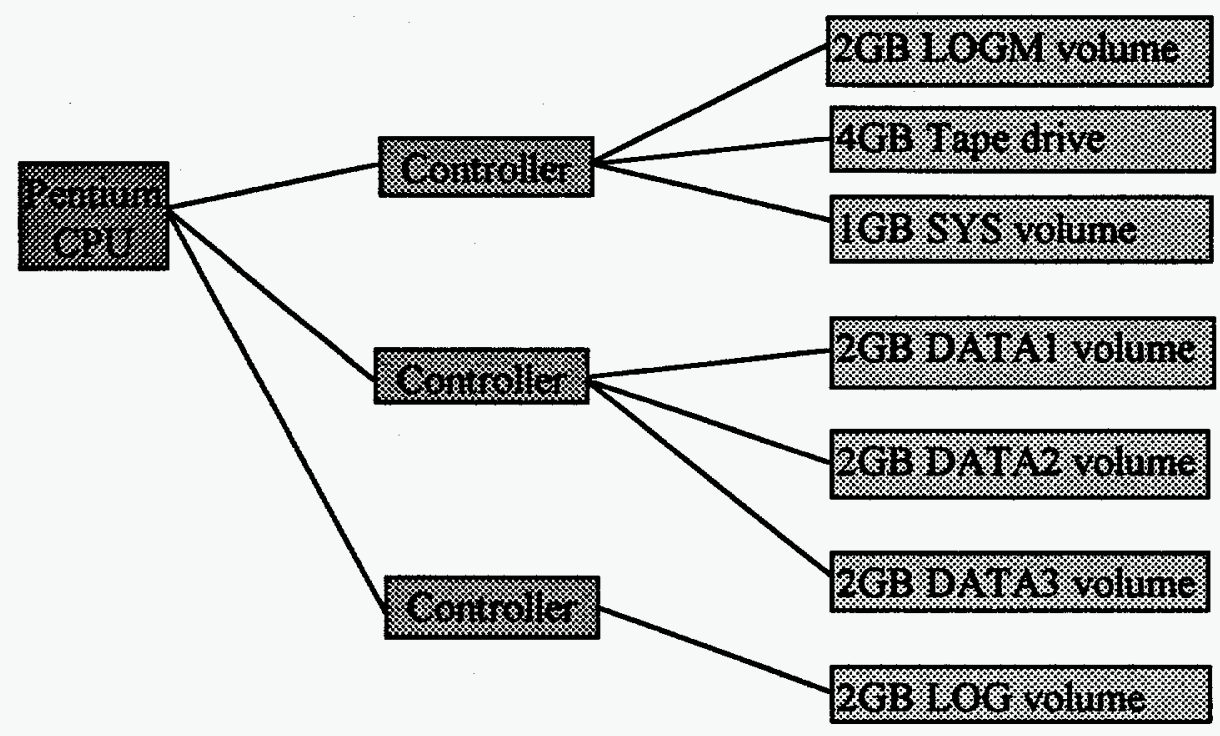

We have multiple controllers for workload distribution and for safety of data (if controller for data disks goes bad, the log should still be intact.) Note the redundant 2GB Log volume. Redundancy of the $\log$ is for mirroring. Explanation: In Sybase, if the log volume fails and it is not mirrored, the database is regarded as corrupt. The result: The current contents of the Log are totally inaccessible AND the database itself is totally inaccessible due to being flagged as "corrupt"!!! To resolve the "corruption", the database must be restored, and the restoration cannot include the current contents of the $\log$ (because the $\log$ is not accessible). Conclusion: If the $\log$ is not mirrored, a failure of the log volume causes the database to be restored back to the last successful backup even though the database itself suffered no failure or corruption.

Useful commands: Volume info comes from load install, volinfo, or rconsole. 
Detailed specifications for DPS/R production database server as installed Sept 1, 1994

\begin{tabular}{|c|l|}
\hline QTY & \multicolumn{1}{|c|}{ DESCRIPTION } \\
\hline 1 & $\begin{array}{l}\text { IBM PS/2 9595 OPT 60MHZ Pentium server (Microchannel bus) with 16MB } \\
\text { ECC RAM, one Fast/Wide SCSI-2 controller card, and 1GB SCSI disk } \\
\text { standard }\end{array}$ \\
\hline 6 & $16 \mathrm{MB}$ ECC RAM modules for a total of 112MB RAM \\
\hline 3 & Hard disk kit \\
\hline 2 & Fast/Wide SCSI-2 controller-cards for a total of 3 \\
\hline 2 & Auto-LANstreamer 32-bit token-ring network cards \\
\hline 1 & IBM DOS 6.1 \\
\hline 5 & $\begin{array}{l}\text { 2.0GB Fast/Wide SCSI-2 hard drives, for a total of 10GB including the } \\
\text { standard 1GB drive }\end{array}$ \\
\hline 1 & $\begin{array}{l}\text { 4/10GB 4mm DAT tape backup drive (4GB standard, 10GB with } \\
\text { compression) }\end{array}$ \\
\hline 4 & 16-bit SCSI cable for Fast/Wide drives \\
\hline 1 & Netware 386 version 3.12, 100 user pack \\
\hline
\end{tabular}

Note: When you run configuration, set up the LANstreamer for 32 bit operation and watch out for an * next to memory allocation. * indicates a conflict between memory allocations in the config. Also, when you put in the tape drive, you need not load drivers, just run configuration.

\section{Software}

- Drivers for Novell implementation of the items listed above IBM drivers SCSI hard drives. NLM is PS2OPT and is in STARTUP.NCF LANstreamer token ring cards --

For IPX, use IBMMPCO.NCF (comes with LANstreamer card).

For TCP IP, use snmp, ibmmpco, tcpip, iptunnel, ipconfig and tcpcon.

The architecture for communications software is, in general, structured as in the following diagram:

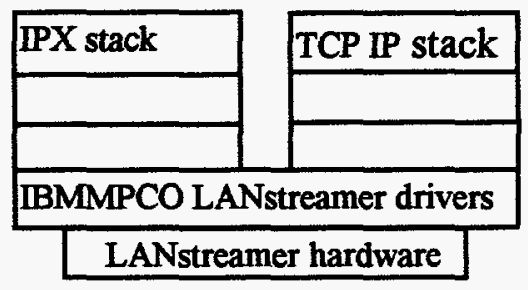

DAT tape backup drive --

To support use of Novell's SBACKUP NLM, the server must have TSA312.NLM, ASPITRAN.NLM, DIBIDAI.NLM and, of course, SBACKUP.NLM. The first two NLMs are started by the AUTOEXEC.NCF (the two must be running before SBACKUP is initiated). SBACKUP will start up DIBIDAI when needed. See special problems associated with backup below. 
To support use of Sybase DUMP utilities, the server must have TAPEDAI.DSK, PHYSADDR.DSK, and DIBIDAI.NLM. All are loaded automatically by Sybase at initiation of backup.

- Sybase 4.2.2 database. Per Sybase Tech Support, various special patches must be included with the Sybase installed software for proper function of the database. The AUTOEXEC.NCF contains the special loads.

- DOS 6.3

- Novell 3.1.2

- Powerchute. The APCSMUPS.NLM is loaded by AUTOEXEC.NCF.

TRICKS AND NOTEWORTHY POINTS

\section{About Sybase}

\section{PROCESS FOR GETTING ALL CUSTOMERS OFF THE DATABASE}

One of the most common complications of the Client/Server environment using Sybase, Novell and Visual basic is identification of customers associated with current logins. We developed the following to address the problem:

- First, your application program (ours is in Visual Basic) must do the connection to the database with a modification of the program name. In Visual Basic, the program name is modified in the Application name variable. Sample code -.-

txtUserName $=$ Trim $\$($ txtUserName)

App.Title = Right\$(App.EXEName, 4) \& " - " \& txtUserName

'The database that is opened is the same as the name as the executable.

$\mathrm{T}=$ "ODBC;uid=dpsruser;pwd=" \& PASSWORDVAR \& ";dsn="

$\mathrm{T}=\mathrm{T}$ \& App.EXEName

Set dpsr = OpenDatabase("", False, False, G_LoginString)

'After making the connection, change the App.Title back so the Windows

'task list will show the application's name

- Next, run an sql to find the connections in Sybase. The following statement does it: use master

go

select spid, program_name from sysprocesses order by program_name go

- Do KILL nn where $n$ is the 'spid' from the list for each user shown, or use customer name shown under 'prgram_name' and call each person and have them disconnect.

- Do

sp_dboption dbname,"dbo use", true

go

checkpoint

go

to shut down the database to customers.

- After doing whatever work is needed, do

sp_dboption dbname, "dbo use", false 
go

checkpoint

go

to return the database to general use.

\section{MOST USED NOVELL COMMANDS AND SYBASE INSTRUCTIONS}

- 'MODULES' at the system prompt shows you what NLMs are running.

- CONFIG shows you the communications configuration.

- CLS clears the screen.

- Down and up of server consists of (at the system prompt) DOWN then EXIT then SERVER.EXE at DOS. SERVER.EXE starts up Novell. Note that server.exe kicks off an invisible NLM (by invisible we mean it doesn't show up when you do the 'modules' command) for the server operating system.

\section{MEMORY AND DISK MANAGEMENT}

- Notes: Sybase sets up segments to allow a layer of controls between the devices and the tables; you can assign a piece of a device (physical name like a dos file name) via a segment and then assign the segment to a table. The assignments allow you to put a portion of a given table onto a given device. Note that what Sybase recognizes as a 'device' is actually a dos file level piece of data storage space. The specification of devices as dos files allows very fine control over utilization of physical resources. In moving the syslogs to lislog device, we are sending the transaction log to the LOG: drive (i.e. volume). I.e., the transaction log table is on the dpsrlis.syslogs segment (created by Sybase automatically for the transaction log table). The segment can be placed on any physical device associated with Sybase (a device is like a dos file).

To do memory management setup in Sybase. Get into an isql session on Desktop DBA. Do

- sp_configure "memory",32000 This assigns 32,000 of $2 \mathrm{~K}$ blocks (i.e. 64,000 bytes) memory to Sybase. Our calculations show Novell should require no more than $30 \mathrm{~K}$ or so of the 112,000 total. $112-30$ is $82 \mathrm{~K}$, but Sybase can not use more than 64K. So set Sybase at $64 \mathrm{~K}$.

- sp_configure "user connections",60 Assigns the number of concurrent users expected. Each user gets 2 connections.! -- one when they connect and another when an ODBC call is used.

- sp_configure "procedure cache", 1 Assign a portion (one percent) of Sybase's allocated memory to stored procedures. Guess 2 per user $x 60$ users $x 5 \mathrm{~KB}$ per stored procedure $=300 \mathrm{~KB}$ and then divide $300 \mathrm{~KB} / 64000 \mathrm{~KB}=1 \%$. New val $=6$.

- go

- reconfigure

- go

- You can list the data from your adjustments by doing select name, value, comments from sysconfigures 
Error handling note: If the transaction log ever gets so full it shuts down Sybase, then you can clear it by doing

$$
\text { dump tran dpsrlis with no_log }
$$

The above will truncate the log and not attempt to write an entry about the log being truncated. If you want to clear the $\log$ and leave a $\log$ entry (i.e. truncate in conjunction with doing a backup), then do dump tran dpsrlis with truncate_only

\section{RUNNING OUT OF LOG DISK SPACE}

Extremely important regarding running out of space on the production database. If you must use dump tran dpsrprod with no_log to clear the log so the system will run, you need to immediately do a dump database dpsrprod to reinstate transaction logging and then enlarge the database with alter database. See page 4-13 of the System Admin. Guide.

Also, you need to get into Desktop DBA (DTDBA) at the isql prompt and run the following sql

use master

go

select a.segmap, a.size from sysusages a, sysdatabases $b$

where a.dbid = b.dbid and b.name = "dpsrprod"

and file the output with the backup tapes. The information is essential to the recovery process since the configuration of fragments (additional space hung onto the database) must be identical on the new logical device (the new disk drive, if you have to replace drives) as it was on the old drives. Otherwise, the restore programs do not know where to put the data.

Also of interest, to drop a device created incorrectly: Do

sp_dropdevice devname or do

drop device devname

then bounce sqlserver by doing shutdown at isql and load sqlsrvr (necessary to get Sybase to free up the device number). Then, at the dos prompt on client machine, do a map $t:=d p s t / w h a t e v e r v o l u m e$ and go to $t:$ whatever path to the device. Do an erase devicename.dat to clear the file off the machine.

Note: You may want to set up an SQL script program to do tuning of the server. Some of the things it might include would be:

sp_configure "user connections", 110

sp_configure "memory", 32000

sp_configure "procedure cache", 6

Also consider including commands to add space to tempdb, and master into this script.

For example,

alter database tempdb on master $=100$

alter database master on master $=19$ 
Before trying to do the backup, at the system prompt, do

modules check to see if aspitran is running; if not, 1) see if dibidai is loaded and if it is, unload it to clear settings; and 2) do load aspitran. load console

Note: The System Administration Guide says to ensure "DSCONSOLE is defined in your environment." The requirement is, although not at all obvious from the Guide, an entry in the SYBENV.DAT file under the sys:Isystem directory on the server. The entry is DSCONSOLE = DPSRSYB. The entry ties the console to the Sybase on the server (doesn't seem like it would be necessary since you're on the server when you're using console). The default entry made by the install routine should be adequate. Note also the Sybase backup routines will dynamically load certain NLMs and device definition files to support the tape device. The files must be present on the Novell server, under the system directory. The files are

physaddr.dsk rumor has it this is empty but Sybase still goes out and looks for it anyway tapedai.dsk dibidai.nlm

The tape is an scsi connected tape drive, and the controller is run by PS2OPT. The PS20PT driver is not sufficient by itself; you must still load the above files.

Non-Tape Daily Backup

- Since the daily backup is a relatively small amount of data, we can employ a backupto-disk method and avoid handling tapes on a daily basis. The process eliminates the complexities of dealing with the CONSOLE screen (CONSOLE need not be loaded) and the need to have aspitran.nlm loaded. Also, dibidai.nlm and physaddr.dsk are not drawn on for the disk dump process. The disk backup process requires a dump device to have been generated. The device is specified under the section of device

specifications. The device is logdump. The device is a 'dump' device, on disk. Dump devices on disk do not have permanent allocation of space (low and high are shown a 0 on the sp_helpdevice display).

- To perform the dump, get to isql and do dump tran dpsrprod to logdump with no_truncate

- Our process for daily backups is to dump every day the entire log as accumulated since the last database dump. Dumping the entire log means not doing a truncate on the log table (the no_truncate option). The result of not doing a truncate is when you must do a restore, you'll need only use the database dump and the latest log dump. You won't need to use all log dumps performed since the database dump. 


\section{BOOTING THE SERVER}

Evolution of the server has produced the following processes for booting the server. These are necessary since several anomalies in Novell and Sybase intervene to prevent a completely automatic boot. Explanations of the specific anomalies are included with the steps driven by each anomaly.

- Doing a cold boot, you will need to enter the power-up password

- The machine will come up in dos. You'll need to enter cdlserver. 312

and server

to bring up Novell. We have removed server from the autoexec.bat to allow more control over things coming up. Having server in the autoexec.bat makes Novell come up and run its autoexec.NCF and allows no user intervention. To bring up Novell without the autoexec getting run, just stay in the root on the $\mathrm{C}$ : drive and enter

\section{c: Iserver.312lserver.exe}

The machine will find the server executable, but will not find the startup.NCF (which resides on the $\mathrm{c}$ : drive in the same directory as the server.exe). Without startup.NCF, the autoexec.NCF will fail due to the ps2opt drivers for disk drives not being loaded. Novell will come up but will run none of the configuration commands on the autoexec.NCF.

- After you do server from the c:Iserver.312 directory, you will need to acknowledge the request for a remote password. As the autoexec.NCF runs, it will come to the load of remote. Press enter to make the remote password default to the supervisor password (we used to put password= on the autoexec line to load the remote, but what that did was put in password= as the new password).

- At the system prompt, enter load sqlsrvr

to start Sybase. Here's one of the anomalies I talked about: If you put load sqlsrvr into the autoexec.NCF, the asynchronous way Novell executes the autoexec makes sql server come up alongside other modules and it crashes.

- Go to a client station, get into Desktop DBA and run scripts to re-gen any stored procedures you have. This is another anomaly -- each time SQL Server comes up, it looses track of the executable for the stored procedures. We can force the system to recompile the stored procedure by making it think the proc has changed; to make it think the proc has changed, just run the script to generate the procedure.

- Lock the Monitor.

\section{About Novell}

\section{SPECIAL CAUTION}

About Net-Prot virus scanner!!! Net-Prot can change the archive bit, so don't have it ever mess with Sybase or files addressed by applications. 


\section{TO USE A CD ROM ON A NOVELL MACHINE}

- Off the machine.

- Plug CD drive to the Centronics connector and boot up.

- Let the machine do configuration and reboot again.

- At system prompt in Novell, do

load c:lcdrom

(you may need to look around and find where the cd rom nim is).

- At the system prompt, do cd volume list

to see what the name of the cd is, then do cd mount volumenamefromabove

- To see the cd rom volume from a client station, do volinfo

The volname for access from a client station is the same as the volumenamefromabove

- To dismount the volume, do cd dismount volumenamefromabove

- Also, you can do unload cdrom to close the driver and unload the cd at the same time.

\section{SAMPLE AUTOEXEC.NCF for IBM SERVER USING SCSI, LANSTREAMER, TOKEN RING}

Following is a sample autoexec.NCF showing many of the options for configuration of disk connections, communications, backup utility support.

FILE SERVER NAME DPSR

IPX INTERNAL NET 10204DB

SET MAXIMUM PACKET RECEIVE BUFFERS $=500$

REM above parameter is for LANstreamer - comment out the above if you're not

REM using LANstreamer coms card. See documentation for other types of cards

REM to see if they say anything about packet receive buffers.

REM Following 4 LINES are per SYBASE setup instructions

SET ENABLE DISK READ AFTER WRITE VERIFY = off

REM and of the 4, the following 3 lines added for System 10

SET IMMEDIATE PURGE OF DELETED FILES $=$ ON

SET FILE DELETE WAIT TIME $=1 \mathrm{MIN}$

SET MINIMUM FILE DELETE WAIT TIME $=30$ SEC

REM Load supporting files for SBACKUP: (LOAD IN ORDER!)

LOAD TSA312

LOAD ASPITRAN

REM LOAD TAPEDAI

REM If load TAPEDAI from command line, then SCAN FOR NEW DEVICES.

SEARCH ADD SYS:SYSTEM/DIBI

SEARCH ADD SYS:SYSTEM/DR

SEARCH ADD SYS:SYSTEMTSA

REM LOAD SBACKUP 
REM The following loads are specified by Sybase Tech Support:

SEARCH ADD SYS:/SYBASE/NLMS

SEARCH ADD SYS:/DPSRINCFS

LOAD AFTER311

LOAD IPXS

LOAD DIRECTFS

MOUNT ALL

REM To get the slot numbers for the below statement and for setup of tcp/ip (below), you can REM just bring up the server and do LOAD IBMMPCO (or token) and it will list valid slot REM numbers Take note of what it offers and use the numbers here for slot number. Also of REM significance,you'll note the frame=ETHERNET_802.3 or token-ring_snap is left off of REM the below loading of IBMMPCO. When the frame type is not spec'd, Novell takes the REM default for whatever type of comms card you are using (TOKEN, IBMMPCO, 3c5x9, REM etc.) and its rev or special config on the present network.

LOAD IBMMPCO SLOT=2 NAME=STREAMER1

REM to use ipx on ethernet, comment out above and uncomment the below

REM LOAD load 3c5x9 slot=3 NAME=STREAMER1 frame=ETHERNET_802.3

BIND IPX TO STREAMER1 NET $=62 \mathrm{~A}$

REM For educ and training $856 \mathrm{rm} \mathrm{108,} \mathrm{make} \mathrm{the} \mathrm{above} \mathrm{line} \mathrm{net}=1$ for 800 use $62 \mathrm{~A}$

LOAD APCSMUPS $-\mathrm{dt}=115$

REM Attempt TCP/IP support: (Load in order!)

REM Requires STREAMS, then CLIB to be loaded. Novell loads them if needed.

REM LOAD SNMP This isn't necessary - Novell will load it if it needs it.

REM If you're using token ring insted of LANstreamer, replace IBMMPCO below with

REM TOKEN or ntr2000, e.g. next line Also, note if you leave out frame type, it takes

REM default load $n t r 2000$ slot $=5$ frame=token-ring msb name=whatevername

REM BIND IPX whatevername net $=62 \mathrm{~A}$

REM Note, the frame type for the LANstreamer is token-ring_snap

LOAD IBMMPCO slot=3 name=streamer 2 frame=token-ring_snap

REM if using ethernet, comment out above and uncomment below

REM LOAD 3c5x9 slot=3 name=streamer 2 frame=ETHERNET_802.3

LOAD TCPIP

BIND IP to streamer2 addr=134.253.122.4 mask $=255.255 .255 .0$ gateway $=134.253 .122 .254$

REM If you move the server to another ring, the 'bind IP' line must be changed.

REM Also must use Sybase INTERFAC editor to change the interfac file to reflect the

REM address change.

REM LOAD IPTUNNEL

LOAD IPCONFIG

LOAD TCPCON

REM LOAD SNMPLOG

REM To set up tcpip on machine with one token ring card: In addition to IPX startup, i.e.

REM LOAD token slot $=5$ or now its load ntr 2000 slot $=5$ frame $=$ token-ring msb name $=x x x x$ REM BIND ipx token net $=62 \mathrm{~A}$ or now its bind ipx $x \times x x$ net $=62 \mathrm{~A}$

REM you also need to do

REM LOAD tcpip

REM LOAD token/ntr2000 slot $=5$ name=dick frame=token-ring_snap msb

REM remember, token-ring_snap is the frame type for tcpip

REM BIND ip dick addr=134.253.122.11 mask=255.255.255.0 gateway=134.253.122.254

REM and to make Sybase work with it, do load sybinst, go to utilities, and interfac editor, go 
REM to ip address and fix it up, do F10 - remember, the interfac editor builds up the file REM correctly from your entry of the tcpip address.

REM this was for 4.2.2 see next line for Sys10-LOAD SQLSRVR -p REM for 4.2.2, we're not doing the -p option - it seems to mess up net-prot below REM re the below being commented - we are starting sybase manually since it trips over REM other modules in the autoexec.NCF when it comes up here.

REM LOAD startserver -f RUN servernameplusDB -k

REM on the above line, put RUN__DPSRDB for the runserver file name

REM LOAD CONSOLE

REM Do not try to load CONSOLE from AUTOEXEC because the SQLSRVR database REM must fully load and initialize first. If CONSOLE tries to load before SQLSRVR is done, REM it will cause various (fatal-)errors. (CONSOLE is obsc. for SYS10 anyway)

REM NEXT LINE FIRES UP VIRUS CHECKER

LOAD NET-PROT

\section{LOAD ROUTE}

LOAD REMOTE

REM LOAD REMOTE PASSWORD $=$ - took off the 'password=' since it put in password= as the pswd

LOAD RSPX

REM LOAD MONITOR -P - $\quad$ for now, don't use the 'P option on MONITOR. It REM appears the -P makes NET-PROT see the cpu as $97 \%$ busy and

REM NET-PROT won't run until cpu is less than like $40 \%$ busy.

REM The -P option is to make MONITOR show the cpu \% utilization

REM y application.

LOAD MONITOR

Notes on autoexec.NCF:

- Max packet recv buff=500 is for the LANstreamer card.

- Enable disk read after write verify = off tells Novell NOT to re-verify by read after write.

- TSA312 and ASPITRAN must be pre-loaded to support Novell's SBACKUP. You need to have TSA312.nlm, ASPITRAN.dsk, TAPEDAI.dsk and DIBIDAI.nlm on the SYS: drive under the SYSTEM directory. The last two of these are called by SBACKUP.nlm and the Sybase backup utility if they are needed. Sybase also draws on PHYSADDR.dsk, so it needs to be under the SYSTEM directory too. All but PHYSADDR.dsk are loaded to the machine by the Novell install process. TSA is Target Service Agent --- it's an nlm, the rest are NLMs and disk drivers to coordinate the hardware.

- The SYS:SYSTEM/DIBI search path is a data file for tape data and SYS:SYSTEM/TSA is a temp directory for use during backup. SYS:SYSTEM/DR contains a special nlm and its workspace.

- The search paths SYS:SYBASE/NLMS and SYS:DPSR/NCFS are for Sybase NLMs and NCFs. AFTER311.NLM and IPXS.NLM and DIRECTFS (a high-speed file handler) are supplied by Sybase. All are loaded per Sybase's instructions.

- MOUNT ALL is a Novell command. It brings up all the disk drives.

- Load IBMMPCO SLOT... loads a driver for the LANstreamer token ring card.

- BIND IPX TO ... sets up the LANstreamer card to run IPX protocall. 
- LOAD APCSMUPS ... brings up Powerchute software and sets the delay time for power kill to 115 seconds after shutdown is initiated.

- Load snmp and all the commands down to load tcpcon do the setup of TCP/IP communications protocal. Load snmp (system network management protocal) brings up token ring components, and load ibmmpco loads the drivers. Load tcpip starts the API level software, and the bind IP to... attaches the API to the correct token ring card. The mask $=\ldots$ and gateway $=\ldots$ specify router (gateway number) and the breadth of routers you want to be recognizing your address (the fewer levels specd for receipt, the wider receiving, more redundancy, you have - the mask produces a string of binary 1 s for an anding operation; the 0 produces zeros; so the anding with the tcpip address strips off the parts of the address where you have a 0, leaving the rest of the levels of the address, and these are the levels that will get your request for receipt). The tcpip address is specd in the bind statement. Iptunnel handles some special communications function (probably routing functions). Ipconfig makes gateways and such equipment available and tcpcon performs communications with gateways. Note the slot number is 3 on the setup of token ring card for tcpip. We are using two separate cards to do tcpip and ipx. Note, under sys: volume, a directory called ETC contains a list used by tcpip to find other machines using tcpip. The list is used in connecting to other machines. All NLMs but ibmmpco are delivered with Novell and are installed by the Novell install. Ibmmpco came on the diskette with the LANstreamer card. Note that so far as Novell is concerned, all setup of tcpip is done in the autoexec.NCF; no net.cfg type file is needed. The only other place tcpip has setup info is in Sybase. Sybase has its interfac file for config info.

- LOAD STARTSERVER -fRUN_DPSRDB -k starts up Sybase. The -f RUN_DPSRDB is a file to tell Sybase where to find MASTER and where to put the ERRORLOG and to max out the number of user connections (the ULIMIT parameter in the file). The $-k$ tells Sybase to kill the backup server when you bring down Sybase with shutdown command.

- For Sybase 4.2.2, it was LOAD SQLSRVR -p (and the -p said 'performance'. i.e., Sybase hogs the machine) to bring up Sybase. In System 10, you can do the same thing, according to the manuals. The LOAD CONSOLE was also for 4.2.2, it is commented out. In 4.2.2, Console (Sybase's console) had to be loaded before you used the Sybase backup tools, but you couldn't bring it up in the autoexec.NCF since NCF commands run asynchronously, so console would come up out-of-sync with the rest of Sybase and crash the machine. Console had to be kicked off later.

- LOAD ROUTE brings up communications software to support IPX via routers.

- LOAD REMOTE PASSWORD= is for rconsole. It says to use the system password and bring the system clear up without an operator entering a password.

- LOAD RSPX is an nlm necessary for rconsole to work on IPX.

- LOAD MONITOR -P loads the monitor. It locks the console keyboard. The -P says to display performance stats.

- Note, CLIB is not needed. It's a library of files to support C programs, we don't use C.

- Note of explanation on tcp/ip: The ARP (address resolution protocol) that's listed when you do 'configure' at systems prompt after loading tcp/ip is a technique for 
routing comms. What happens under $A R P$ is, the first time one station send a message to another under tcp/ip, the message going out (first time) is a query message asking the sendee to send back its tokenring/ethernet or whatever hardware address. Once the requester station gets this info, it starts normal comms with the other machine. To do normal comms, it sends out packet with both the tcpip and the tokenring/ethernet address. The tcp/ip address is used by routers to get the message down to the last router before the station. The router puts the packet onto the LAN with only the hardware address. If the tcp/ip address were used clear down to the workstation, each workstation on the LAN would have to suck each tcp/ip packet clear up to the cpu so the software could look at the tcp/ip address and see if it's for that station (the token ring card doesn't know anything about tcp/ip - tcp/ip is purely at the cpu level). Having each packet of tcp/ip sucked into the cpu on each station, the load on each stations cpu would be enormous if very much tcp/ip comms were going on. By having the router put the packet onto the local network addressed by the hardware address (token ring address) each station gets all packets only into the token ring card. The token ring card feeds only the packets for the given station up to the cpu.

- The following tells you the type of changes needed if you put in an ethernet card. These entries make the LANstreamer card get ignored and the ethernet card get used For use in on an EtherNet network, REM the two lines for loading token and binding it:

load TOKEN slot $=5$

bind IPX to TOKEN net $=62 \mathrm{~A}$

Then unrem the following two lines. (Training room 108, bldg 856, needs this change)

rem load 3c5x9 slot=3 frame=ETHERNET_802.3

rem bind IPX to $3 c 5 \times 9$ net=1 <-----get this number from lan manager or maybe by doing userlist/address to see addresses of current users

For the LANstreamer card, the changes are, you comment out the

LOAD IBMMPCO SLOT=2 NAME=STREAMER1

BIND IPX TO STREAMER 1 NET $=62 \mathrm{~A}$

and put in

load $3 \mathrm{c} 5 \mathrm{x} 9$ slot=3 frame=ETHERNET_802.3

bind IPX to $3 c 5 \times 9$ net $=1$

\section{AUTOEXEC.NCF INSTALLATION SITUATIONS}

If the system prompts you to build the autoexec.NCF and startup.NCF, build them per above. If the right parameters are not there to run disk drives and other devices, the install may not ask you to build autoexec and startup (and it will fail to save anything you have done!). Instead, you will need to install the drivers listed on page 36 of the Install and Upgrade Guide. Drivers needed are LOAD PS2OPT SLOT $=1$ (or whatever the slot is -if it was under "built in features" when you viewed configuration, you'll need to put SLOT $=99$ ) then do load c:lserver.312linstall to bring the install functions back up. See page 41 of the Guide. 
When you install Novell, make the block size on disks 16K (essential for performance -also impacts memory requirements; apparently Novell keeps a table of addresses in memory, and the smaller the frame, the bigger the table, and the difference is huge!).

\section{NOVELL 4.X SETUP - NOTES}

- In Novell 4.x, you'll be prompted for selections to enter for drivers and configuration parameters. You need to select:

- PS2SCSI for the disk driver

- NTR2000_LAN for token ring coms

- Set tcpip address to 134.255 .122 .153

- Put in token ring card slot number and no entry for token ring card num. For ethernet, select 3c5x9.

- Create partitions. Take all defaults, do escape and F10.

- Create volumes. Take defaults including block size.

- Put in 10 user license disk when asked for it

To install LANstreamer communications. See page 2-11 in the LANstreamer book. Use LANstreamer option diskette in the server's A drive and do load a: Inovell netwarelibmmpco slot $=2$ name=streamer Note - if you have a token ring card (not LANstreamer), do load token slot $=\mathrm{n}$ name $=$ token

and be aware that token is on the server from Novell install; you don't need an option diskette for token ring.

and then

bind ipx to streamer net $=62 \mathrm{~A}$

(Notes: Expect some error messages when you load ibmmpco. Use of the name streamer is optional; you can use another name, or, you can leave out name $=\mathbf{x x x}$. If you leave it out, on the bind statement, put bind ipx to ibmmpco net $=62 \mathrm{~A}$. A name is required if you have more than one token ring card -- the specification of slot numbers to spec which slot doesn't work well. Some other NLMs were required for earlier Novell -- patchman.nlm and lslenh.nlm -- but these are not needed for 3.12. About the 62A ring id: 62A was assigned automatically by the first IPX machine to come up under our router. All subsequent IPX machines brought up under our router and performing functions requiring broadcast of a server IPX address, must use the same ring id.)

Note: If you're using token ring communications (not LANstreamer) then you can skip this paragraph (i.e., skip the copy of communications card drivers to the system directory) since the token driver is included with the Novell installation, and no copying from an option diskette is required. If you're using LANstreamer or other comms that are not on the Novell disks, then take the LANstreamer option diskette to a client station. Login dpsr/supervisor. Do a map like the following (you may need to use something other than $\mathbf{x}$ : for the drive if your $\mathbf{x}$ drive letter is already assigned to something):

map $\mathbf{x}=$ dpsr/sys: and then 
Then do

copy a:Inovellnetwarelibmmpco. ${ }^{*} x:$ lsystem

attr msm31x.nlm -r

and also do

attr tokentsm.nim -r

and

attr ethertsm.nlm $-\mathrm{r}$

Then copy the three from the A: drive to $\mathrm{x}$ :lsystem, go to the $\mathrm{x}$ : lsystem directory and do attr ms3 $1 x . n l m+r$

and

and

attr tokentsm.nlm $+r$

attr ethertsm.nlm $+r$

Important note about setting up autoexec.NCF:

If you have a fatal flaw in the AUTOEXEC (that won't let the server load enough for you to clean it up before DOWNing and restarting), you should be able to start the server by running SERVER -NA which stops autoexec.NCF from running. If SERVER -NA won't work, try this:

Get out to DOS (from system prompt, enter EXIT)

Run SERVER.EXE in a way that keeps the disk drivers from loading, either by:

- Calling SERVER from a directory other than the one where STARTUP.NCF resides (i.e. go to root and do c:lserver.312/server.exe --- this makes the machine kick off server.exe, but it won't be able to find STARTUP.NCF, and will fail when it tries to run autoexec.NCF), or

- Bashing STARTUP.NCF so disk drivers (ps2opt) won't load, then run SERVER

The result is that the Novell loads but cannot find the disk drivers. When this happens it cannot find anything on the Novell partitions where AUTOEXEC.NCF resides, and will fail to execute AUTOEXEC.NCF (note that autoexec.NCF always gets kicked off by server.exe, but with the commands in startup.NCF not found by server.exe, the autoexec.NCF will fail). Now it's dumb and prompts you for everything. Use this to your advantage by loading only what you need to fix the problem (load c:Iserver.312lps2opt, mount SYS, load edit or install or whatever to get to an editor for autoexec.NCF). Once all is well you can exit to DOS and re-run SERVER.EXE from its own directory, let it find the correct disk drivers in STARTUP.NCF

Note: It's a good idea to test all commands before you put them into autoexec.NCF. Test anything you want to put into AUTOEXEC.NCF by doing it first at the console prompt. You can really hurt yourself by putting untested commands in the AUTOEXEC.

Generally, the contents of the autoexec.bat for a NOVEL server on DOS will look something like: 
@echo off

prompt \$p\$g

path $=$ c:Idos

set temp $=c$ :Itemp

:begin_server

c:

cd c:Iserver. 312

@echo Starting Novell...

server

:end_server

c:

cdl

Notes about autoexec.bat:

- The autoexec.bat resides in the DOS partition. See above for notes on autoexec.NCF, server.exe and startup.NCF.

- Basically all it does is set the path, set TEMP, and launch server.exe.

\section{LOGIN SCRIPTS - a primer:}

- Note about login scripts: If you log into a master server and it attaches you to other servers, the other server's script never gets run. Only the master's script gets run.

- The login script is the only place where passthrough connections to other machines are handled. The 'attach' command in the login script makes the connection to the other servers and handles password synching. No other setup is required on the server to get passthrough connections or the associated password synching performed.

- About \# commands: The \# in a script is a call to an executable. The executable is sent to the workstation and executed there. Executable to be run with \# must reside in the PUBLIC directory. Other execs in the script are just run by Novell on the server, like fdisplay for example runs a program to send a display to the workstation, but the executable runs on the server. Executables shipped to the client station by the \# could also be run from the workstation. You would need to just enter, for example, the statement wsupdate at the dos prompt to run the wsupdate program.

- sample use of fdisplay is fdisplay f:Ipublicltest1.doc

which, interestingly enough, uses your $f$ : drive mapping to see what it's going to display

- In the login script you need to include map ins s16:=sys: Ipublic

Here, the lack of anything before the sys: says to use the present server, and the ins says to map without overlaying other mappings, 16 says to map to the last available drive, S\# says to put in the path (see your notes in docu.bat). You need to have a mapping to public directory so it can find programs like NCOPY when you do \#NCOPY

The map command 


$$
\text { map *1:= -......-.- }
$$

the * says to map to the 1 st drive after the ones done by dos.

$$
\text { map p:=sys: Ipublic }
$$

does a 'hard' mapping to $p$ : drive

- The command

\#NCOPY source destination

sends an executable to your workstation to copy the source file to your destination file.

- The command

\#wsupdate /f-sys: Ipubliclwsupdate.cfg

sends you and executable to do whatever updates are specd in the parameters file called wsupdate.cfg

- The command

$$
\begin{aligned}
& \text { if login_name ! = "xxxxxx" then } \\
& \text { end }
\end{aligned}
$$

is an if statement to test for the value of your login name

- Another if type statement

$$
\begin{aligned}
& \text { if not member of "developer" then } \\
& \text { end }
\end{aligned}
$$

to test for membership in a group.

- The command

pause

makes the server stop during login till the person hits a key.

- To attach to other servers, do

$$
\text { attach servername/\%Login_name }
$$

Here, the \% is a concat command, the field name is Login name. The filed called Login_name is a pre-defined parameter set up by Novell. You need also to do map m:=servernamelsys: Iccmail

or

$$
\text { map ins s16:=servernamelsys:lccmail }
$$

to get the mapping for the attached servers. Note that you need 2 mappings if you want to have a known drive letter and to have the sever in your path too.

\section{NOTES ON RECOVERY WHEN NOVELL WON'T BOOT!!!!!!!!!!!}

About the Sybase tendency to crash with comm line noise: It stops Sybase and won't let you do any isql or unload sqlsrvr. So, if Sybase is stopped to users and the Sybase message screen has no indication of the error (which indicates it's this com noise problem) dont try - to do an unload sqlsrvr or load isql, instead, just do a down of Novell and reboot the server. If you have tried to unload sqlsrvr and so have locked up the whole server (Novell and all), then you'll have to cut power to reboot. When you come up, you may observe Novell asking for the server name and the IPX connection id; if so, you've got a trashed fat table on the SYS: volume. (Novell keeps 2 copies of the fat table and 
compares them when it's starting up; if they are different, Novell won't mount the volume and since the volume isn't mounted, Novell won't find the autoexec.NCF and since it can't read the autoexec.NCF, it will ask for the server name and IPX port just like you were bringing up Novell from a diskette.) To fix the fat table, you need to boot the machine, do the cdlserver.312 and do server. Let Novell come up and give it the name of the server and a port number. When Novell gets to the system prompt, do load c:Iserver.312lvrepair and when vrepair comes up, select the option to fix a volume and give it SYS:. It should be ok after this.

\section{DATA COMMUNICATIONS UNDER NOVELL AND TCPIP}

The greatest source of downtime to DPS/R has been data communications problems. The following provides a bit of background on the used of the database ping and fileserver ping commands. Also covered is one type of data communications failure involving loss of information by a router. The logic used by a tcpip router is described and one solution we have used is provided.

- When you do wdbping (do it from file run in Windows) you will see a list of sqlserver database names. The names are associated with entries in the win.ini file. The name identifies whether the connection is to use tcpip or ipx (in the wdbnovdb or tc) and the address of the server. Also identified is whether the connection is as a database user or an administrative login to Sybase (identified by the port connection number -- e.g. $83 \mathrm{db}$ or whatever). If wdbping doesn't find dblib and netlib in memory, shell to dos and do a sybclenv.bat.

- To do a ping from server to a client station: Get to system prompt, do load ping and then fill in the tcpip address, hit enter a couple of times, and then press escape. The server will start sending packets to the client you spec'd and will show the counts of packets sent and the responses received. Hit escape again to exit the ping program.

- The above process is used to correct one of the problems the routers have: In tcpip, the first packet coming in to the server is identified only by the tcpip address -- all stations on the ring pick up the packet and see if it's for them or someone else. When the server responds, the router captures the tcpip address and network adapter (token ring) card i.d. and saves it; thereafter, when the router puts a packet onto the ring for the server, it puts on the token ring address so only the server's token ring card will pick up the packets, and the other stations on the ring won't be wasting time bringing the packets into memory and interrogating the tcpip address (in tcpip protocal level software). Now, the problem is the router occasionally will forget or scramble the token ring address number and will put packets on the ring that are not identified correctly. When this happens, clients can no longer get to the server. The router thinks it has correct token ring address info from a previous packet, so is putting on token ring address and leaving off the broadcast tcpip info, so the server is getting bad token ring addressing and no tcpip broadcast info, so the packets don't get into the server at all. To reset the router token ring data, you can go to the server and do a 
ping of the client station who can't get to the server. When the server sends out the packet to the client, the router will capture the correct tcpip-to-token ring cross reference and communications between the server and the client stations should be restored.

\section{About Visual Basic}

\section{DATE-TIME SELECTS USING VB AND SYBASE}

Where a date-time value has been used as a key to database records: Complications of generating sql to use the date-time stamp from one record to select others.

- The problem is Visual Basic's date time field will not contain a date-time value of the accuracy needed to do a select against the Sybase data. The lack of accuracy causes a mismatch on field values.

- Solution is to do a select for values between two date-time stamp values.

- First, separate the input date-time stamp into a date number and a time number using VB's datevalue and timevalue functions. Call date $D$ and the time $T$.

- Second, Convert the time portion (T) to a string, extract the first 12 positions, and convert back to a number. Call this A

- Add .0000000001 to the number. Call this B

- Write select statement to select for dates between D + A and D + B

\section{VB REFUSES TO OPEN A TABLE}

Bug resolution: If you find VB refuses to open a Sybase table using ODBC, check the table for a unique key. VB will refuse to open a table not having at least one unique key.

\section{MOUSEPOINTER TIP}

If you're using modal forms, you can save yourself some grief in changing appearance of mouse pointers by using screen.mousepointer instead of form.mousepointer.

\section{LAN ADDRESSING -- ROUTER CONSIDERATIONS FOR SYBASE}

In IPX communications, LAN addresses in WIN.INI are dependent on the segment of the LAN you're on. Apparently this is caused by presence of a router in the communications path.

- If you're on the same segment as the server, the following will work

DSQUERY=WDBNOVSP, servername

- If you must go across a router, the above will not work. You must use

DSQUERY=WDBNOVSP,internal_network_id,1,data_server_tag

where the internal_network id is set up by you during Novell install (in autoexec.NCF). 


\section{DESKTOP DBA TIMEOUT DUE TO DB SIZE}

If you try to do a create database using Desktop DBA and the size is over about $10 \mathrm{meg}$, Desktop DBA causes a timing error and Sybase backs out the create. Solution: Dont' use DTDBA to create databases over $10 \mathrm{meg}$. Use isql on the console or rconsole.

\section{NUMBER OF USER CONNECTIONS}

Remember to set your user connections in Sybase to twice the number of users you expect on the system. One connection is used at login and the other when SQL is run.

\section{DOS BCPS AND DATE FIELD PROBLEMS}

DOS version of Sybase BCPs: DOS BCPs will choke on blanks in date fields; it doesn't know to put in 1900-01-01. DOS version will put null if field is ended with a comma and database is set to accept nulls. Also, be sure to use yyyymmdd format for dates - it crosses all Sybase communication.

\section{BCP SPEED}

- To make BCPs go faster, first delete indexes. Sybase can load files and rebuild indexes faster than loading data and building as-you-go.

- Be sure to set select into/bulk copy on in sp-config. It stops generation of transaction $\log$, BUT if there are indexes being written as bcp runs, Sybase still builds log entries.

\section{BCP DATA ERROR LOCATION}

Though bcp error reporting is nearly useless, bcp does allow you to home in on an error with a binary search. Use the -Fnn (first record to be loaded from the input data set) and Lnn (last record of the data set to be loaded) and specify a range of data to load. See whether the error is in the range and modify the range to home in on it. Once you locate the erred record, you must study it character-by-character to find the data problem.

\section{SYBASE SENSITIVE TO COM-LINE NOISE}

Sybase com line noise: It may have improved with System 10, but in 4.2.2, unplugging a comms connector on the back of the server and plugging it back in would crash Sybase. Check this out if your have strange crashes.

\section{SYBASE VARCHAR}

In Sybase, writes of varchar field automatically drop an training blanks. Don't bother stripping trailing blanks in your code. 


\section{SYBASE DAT TAPE BACKUP}

If you're using a DAT tape for backup in Sybase, you must have aspitran loaded before the backup will run. If it is in the autoexec.NCF, it will often still not load and you'll get and error on the dump saying dibidai can not be loaded. Check for aspitran before beginning backup where tape is driven by PS2OPT. Check by doing modules.

\section{SUBROUTINE CALLS FROM DYNAMIC LISTS}

Dynamic subroutine calls can be done in VB without a huge case statement by using the drag event. Set move mode to manual on a button and you'll get a move event you can put your subroutine in.

- Locate the control to be run by looking in the forms collection using for $I=0$ to controls. count -1

If typeof me.controls $(\mathrm{I})$ is commandbutton then

if me.controls(I).caption = "literal you're searching for" me.controls(I).drag 1

endif

me.controls(I).drag 2

endif

next I 


\section{REFERENCES}

The following are suggested as the more compact sources of additional information:

1. Chris McCartney, Cristina Nitescu, SYBASE SQL Server Troubleshooting guide (SQL Server 4.0.1 through 10.0.1), Emeryville, CA, 1994.

2. Microsoft Corporation, Microsoft Visual Basic Programmer's Guide, Redmond, WA, 1993.

3. Novell, System Administration Novell NetWare 3.12, Provo, UT, 1990.

4. Sybase Server Publications Group, SYBASE SQL Server Reference Mamual, Vol. 1. Emeryville, CA, 1994. 


\begin{tabular}{lrl}
\multicolumn{3}{l}{ DISTRIBUTION: } \\
1 & MS 1012 & E. E. Saucier, 3050 \\
5 & 0899 & Technical Library, 4414 \\
1 & 0805 & D. G. Heckart, 4911 \\
1 & 0181 & R. K. McIntire, 7401 \\
2 & 0100 & Document Processing, 7613-2 \\
& & For DOE/OSTI \\
1 & 0944 & N. L. Freshour, 7901 \\
1 & 9018 & Central Technical Files, 8523-2 \\
1 & 9116 & D. J. Wallace, 8534 \\
1 & 0200 & D. R. Stimak, 10200 \\
1 & 0205 & L. D. Rathjen, 10200 \\
1 & 0203 & L. V. Miranda, 10203 \\
1 & 0204 & R. R. Baird, 10204 \\
1 & 0204 & A. L. Hardeman, 10204 \\
1 & 0204 & M. J. Rouse, 10204 \\
1 & 0202 & C. E. Wenk, 10206 \\
1 & 0202 & C. Schneeberger, 10210 \\
1 & 0221 & D. L. Palmer, 10220 \\
1 & 0201 & L. D. Ellis, 10230 \\
1 & 0201 & C. H. Reeder, 10240 \\
1 & 0202 & G. L. Record, 10250 \\
1 & 0113 & C. H. Duus, 10505 \\
1 & 0619 & Print Media, 12615
\end{tabular}

